\title{
Assessing Material Densities by Vibration Analysis And Independent Component Analysis
}

\author{
Hajar Razaghi $^{1}$, Reza Saatchi ${ }^{*}$, Amaka Offiah ${ }^{3}$, Derek Burke ${ }^{2}$, Nick Bishop ${ }^{3}$ and Santosh Gautam ${ }^{1}$ \\ ${ }^{1}$ Faculty of Art, Computing, Engineering and Sciences, Sheffield Hallam University, Sheffield, United Kingdom \\ ${ }^{2}$ Sheffield Children's Hospital National Health Service Trust, Sheffield, United Kingdom \\ ${ }^{3}$ Department of Human Metabolism, University of Sheffield, Sheffield, United Kingdom \\ Received 1 December 2012, Revised 20 March 2013, Accepted 16 April 2013, Available online 2 May 2013
}

\begin{abstract}
The aim of this study was to investigate vibration analysis and independent component analysis (ICA) to assess the density of multiple materials making up a single structure. Density is important as it reveals information about physical properties of materials. The density of a single material can be determined from the relationship between its mass and volume. However, when a structure consists of multiple materials, identification of their individual densities from the structure is complicated. Vibration analysis is a technique that reveals information about an object's physical properties such as its density. The investigation was carried out using a plastic test tube filled separately with three liquids of known densities; water, Chloroform and Methanol. Vibration was inducted into the tube, through an electronic system that produced a single impact at a predefined location on the tube. The resulting vibration signals were recorded using two vibration sensors placed on the tube. A signal source separation technique called ICA was used to obtain the vibration effects of the liquid and the tube. The power spectral densities (PSD) of ICA extracted vibration signals were examined. The frequency of the largest peak in the PSD was related to the liquid's density under test. The study indicated that vibration analysis may be effective in assessing materials' densities in a structure that contains multiple materials, however a larger study is needed to explore the findings.
\end{abstract}

| Density assessment | Vibration analysis | Independent component analysis |

( 2013 Ibnu Sina Institute. All rights reserved. http://dx.doi.org/10.11113/mjfas.v9n3.96

\section{INTRODUCTION}

The density of materials is an important feature of their physical properties. It is a measure of the amount of a material's mass in relation to its volume. Density is often used to identify and classify substances. It is usually expressed in grams per cubic centimetres $\left(\mathrm{g} / \mathrm{cm}^{3}\right)$. The density of an object that is made from a single material can easily be determined. However, some objects such as bone have a complex structure making their density analysis complicated.

Materials with different densities vibrate differently when they are subjected to an applied force. The form of force inducing the vibration can be different. It can be an impulse, where the applied force has a very short duration. An example of this is when a hammer impacts an object. Alternatively, it can be continuous in nature, for example when a vibration motor is attached to the object under test. In the former, the vibration of object decays with time and the decay rate is an important feature of the object's physical properties. In the latter, the vibration of the object will be on-going and the vibration of the system that induces the vibration and the vibration of the object under test interact, creating a more complex overall vibration signal.
Vibration analysis has been used extensively to better understand the physical behaviour of materials and to detect defects in their structures [1-3].

An issue that complicates vibration analysis is that when the object consists of multiple elements of different densities, these elements vibrate differently and thus the vibration signals recorded are a mixture of vibrations from each element in the structure. This requires that the vibration signal attributed to each element be separated from the recorded signal mixtures.

Signal source separation is a technique that is used to explore signal components in mixtures emanating from independent sources. A well-known signal source separation technique is independent component analysis (ICA). ICA is an extension of the Principal Component Analysis (PCA)that not only de-correlates, but it can also deal with the higher-order statistical dependencies [4, 5]. Bell and Sejnowski [6] proposed an information-theoretic based ICA algorithm that uses an unsupervised learning rule. It finds a linear transformation within the data to make the separated signal components as statistically 'independent' as possible. The technique does not need $a$ priori knowledge of the physical location or the configuration of the sources, and unlike PCA, it does not require the distribution of the signal sources to be orthogonal. However for it to function correctly, the signal sources must be statistically independent and the 
distribution of not more than one source should be Gaussian.

The use of signal source separation in analysing vibration signals has been investigated in a number of studies. ICA has been applied to gear vibration measurements [7]. It has been shown that the individual gear and pinion vibrations cannot be separated using the blind separation algorithm, but the learning curve of the updated parameter can be used to detect impulsive and random changes in the data. ICA has been used to separate vibration signals into contributions of periodic, random stationary and random non-stationary sources [8]. The relation between the vibration modes of mechanical systems and their modes computed through ICA were investigated [9]. For free and random vibrations of weakly damped systems, a one-to-one relationship between the vibration modes and the ICA modes was demonstrated.

In a study, signal source separation was used to detect the influence of one motor from another placed on a work bench [10]. Temporal signals from accelerometers or microphones were recorded. The results indicated that the approach could be successfully applied to vibration analysis.

The outline of this paper is as follows. A brief introduction to ICA is provided in section 2; the experimental methodology used in this study is explained in section 3; results are presented in Section 4 followed by our conclusions in section 5 .

\section{INDEPENDENT COMPONENT ANALYSIS}

The concept of ICA for a situation involving two signal sources $\boldsymbol{S}=\left[\begin{array}{l}\boldsymbol{s}_{1} \\ \boldsymbol{s}_{2}\end{array}\right]$ is illustrated in Fig.1. The mixtures $\boldsymbol{X}=\left[\begin{array}{l}\boldsymbol{x}_{1} \\ \boldsymbol{x}_{2}\end{array}\right]$ are generated by the operation

$$
X=A S
$$

where $\boldsymbol{A}=\left[\begin{array}{ll}a_{11} & a_{12} \\ a_{21} & a_{22}\end{array}\right]$ is the mixing matrix.

The aim is to estimate an unmixing matrix $\boldsymbol{W}=\left[\begin{array}{ll}w_{11} & w_{12} \\ w_{21} & w_{22}\end{array}\right]$ which in turn enables an estimate of the original signal sources $\boldsymbol{U}=\left[\begin{array}{l}\boldsymbol{u}_{1} \\ \mathbf{u}_{2}\end{array}\right]$ to be obtained by,

$$
\boldsymbol{U}=\boldsymbol{W} \boldsymbol{X}
$$

The ICA algorithm proposed by Bell and Sejnowski [6] was used in this study. This algorithm is performed by the following steps.

i) The unmixing matrix $\boldsymbol{W}$ is initialised to an identity matrix.

ii) The signal sources are estimated and then they are transformed by a non-linear transfer function. For a sigmoidal transfer function (used in this study), the resulting signals $(\boldsymbol{Y})$ are expressed as,

$$
\boldsymbol{Y}=\boldsymbol{g}(\boldsymbol{U})=\frac{1}{1+e^{-U+\omega_{o}}}
$$

where $\boldsymbol{\omega}_{\boldsymbol{o}}$ is a vector of bias weights and it is initialised to a zero vector.

iii) The non-linearly transformed signals ( $\boldsymbol{Y}$ ) are processed by a learning rule that maximises their joint entropy (i.e. it minimises their mutual information). This is achieved by changing the weight matrix by the amount $\Delta \boldsymbol{W}$, where

$$
\Delta \boldsymbol{W}=\left[\boldsymbol{W}^{T}\right]^{-1}+(1-2 \boldsymbol{y}) \boldsymbol{x}^{T}
$$

and the symbols $T$ and ${ }^{-1}$ represent matrix transpose and inversion respectively. The change in the bias weight is expressed by,

$$
\Delta \omega_{o}=1-2 y
$$

iv) The ICA algorithm is trained by repeating steps (ii) and (iii). After each iteration, the unmixing matrix $\boldsymbol{W}$ is updated by $\Delta \boldsymbol{W}$ until convergence is achieved. The convergence is achieved when the rate of change falls below a predefined small value (for example 1.0 $\times 10^{-6}$ ). The rate of change is computed by squaring the difference between corresponding elements of the unmixing matrix before and after each iteration and then summing the values.

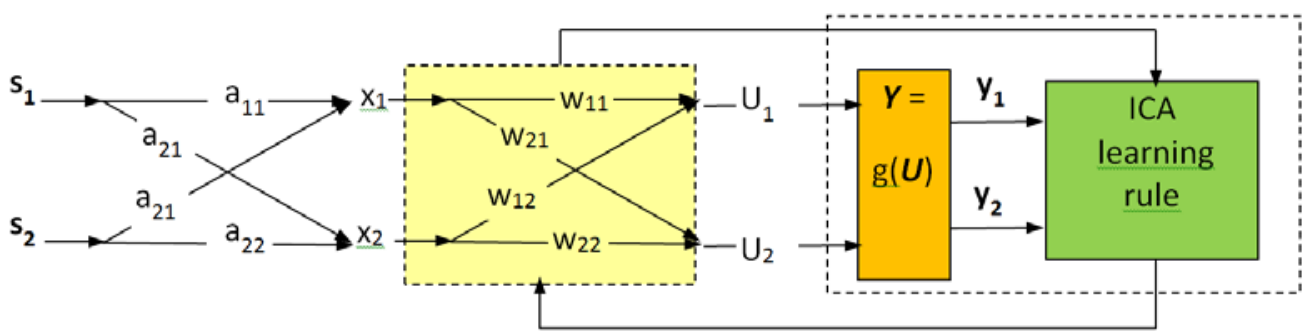

Fig. 1. A block diagram of ICA operation 


\section{METHOLOGY}

An experiment was devised to investigate the effectiveness of vibration analysis and ICA in assessing the densities of multiple elements in a structure. For this purpose, vibration signals were recorded from a plastic laboratory test tube (capacity $30 \mathrm{ml}$ ), when it was empty and when it was individually filled with three liquids of known densities(water, Chloroform and Methanol). Figure 2 shows the experimental set up.

The bottom part of the tube was held in a vice. The force to produce the vibration was provided by a modified solenoid that was connected to a computer through a suitable drive circuitry. The magnitude of the applied force was controlled via the computer. The induced vibration signals were recorded using two piezoelectric sensors (type CM-01B).CM-01B was chosen because it is a lightweight sensor with high sensitivity (typical $40 \mathrm{~V} / \mathrm{mm}$ ) designed to pick up vibration signals, while minimizing external acoustic noise. The impact point and sensor locations were marked by a marker to be identifiable during the experiment (see Figure 2).

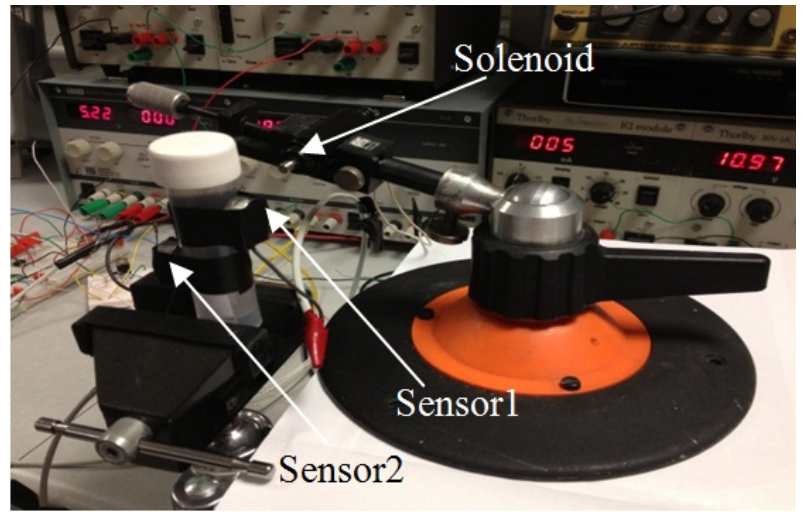

Fig. 2. Experimental set up

It was ensured that the magnitude of the applied force was consistent throughout each test and that the force was applied to the same point on the tube. The vibration signals were recorded using a National Instrument data acquisition system. The signal sample rate was 100,000 samples per second. The tube was exited 20 times to allow the consistency of vibration signals to be examined.

The experiment was repeated when the tube was separately filled with (i) water (ii) Chloroform (iii) Methanol. These liquids were chosen because their known densities are significantly different.

The recorded vibration signals were initially processed by ICA. Then the power spectral densities (PSD) of the resulting extracted components were estimated using the periodogram approach. The periodogram used the Goertzel algorithm.

The relationship between the frequency associated with the highest peak in each PSD and theknown density of the liquid was investigated.

\section{RESULTS AND DISCUSSION}

Figures 3 and 4 show the averaged (over 20 signals) vibration signals together with the corresponding ICA extracted components from sensors 1 and 2 respectively when the plastic tube was empty.
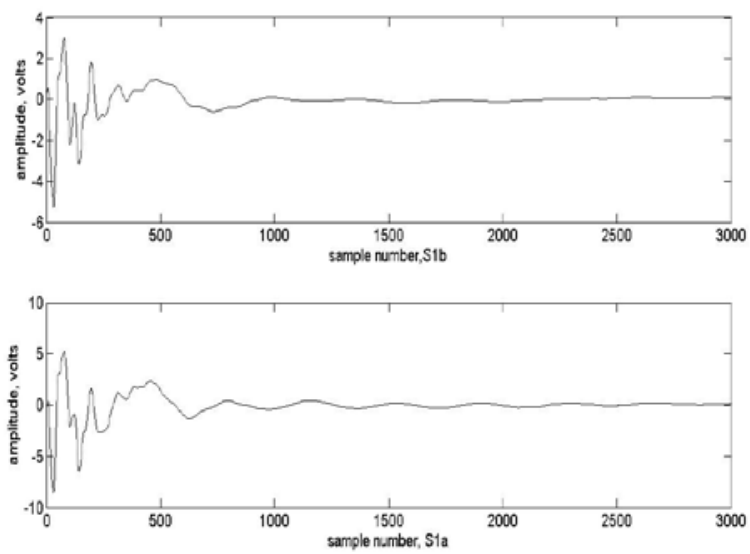

Fig. 3. The averaged vibration signal from sensor 1 before ICA (top) and its corresponding ICA component (bottom)for the empty test tube.
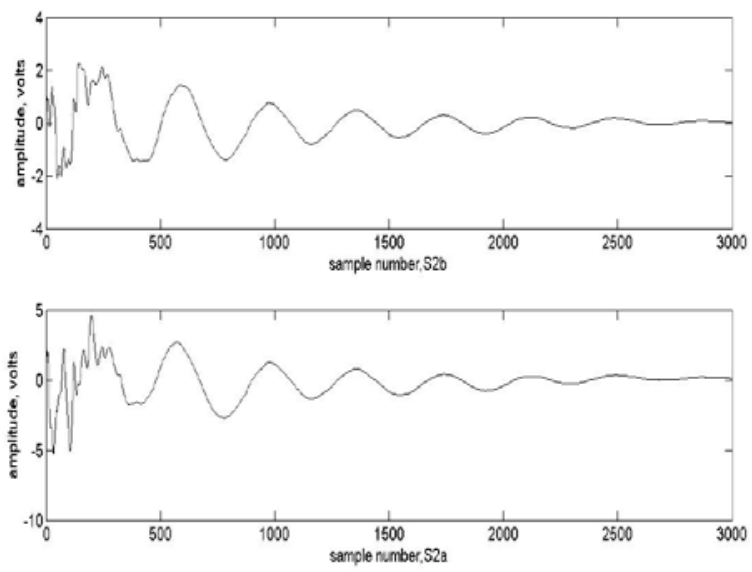

Fig. 4. The averaged vibration signal from sensor 2 before ICA (top) and its corresponding ICA component (bottom)for the empty test tube.

Figures 5 and 6 show the 20 abutted vibration signals recorded from sensors 1 and 2 and their respective ICA extracted components when the plastic tube was empty.

ICA performed more effectively when it processed the vibration signals that consisted of the 20 trials abutted together. Therefore, all analyses reported in this paper are based on the 20-abutted signals. 

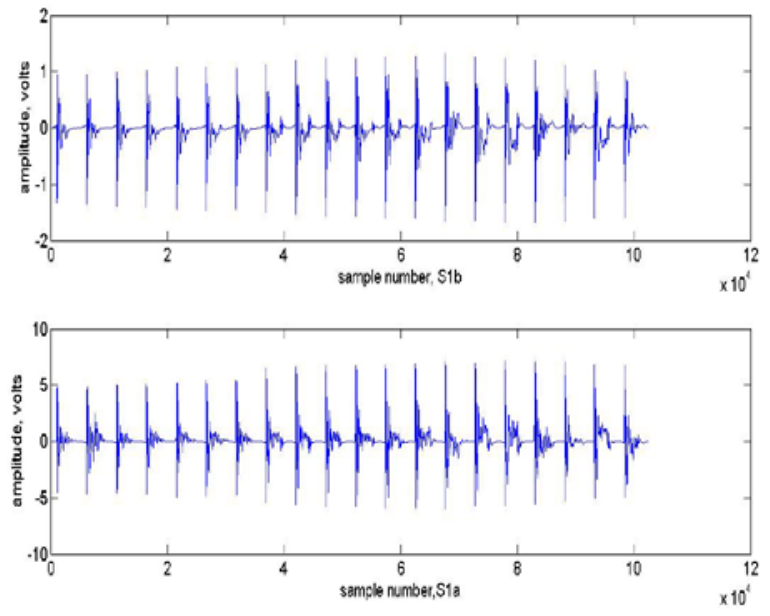

Fig. 5. Twenty abutted vibration signals recorded from sensor 1, before ICA (top) and the corresponding ICA component (bottom) for the empty test tube.
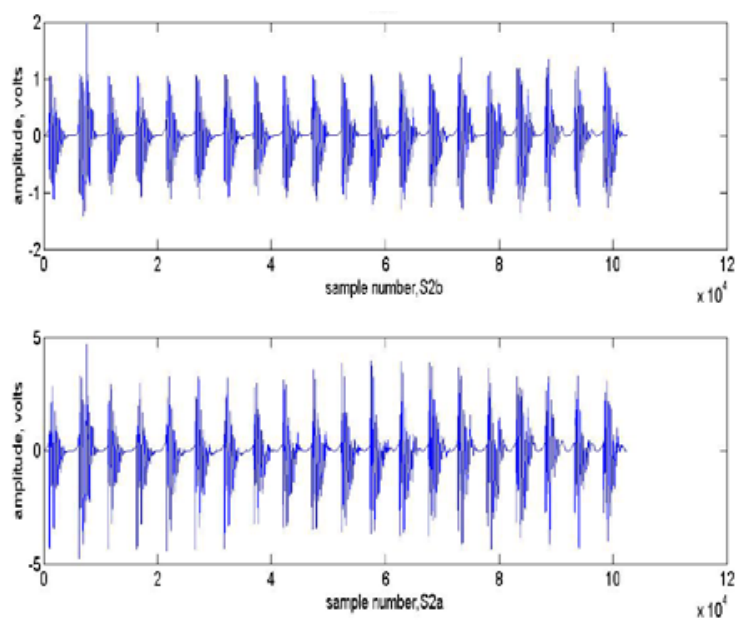

Fig. 6. Twenty abutted vibration signals recorded from sensor 2 before ICA (top),the corresponding component ICA (bottom) for the empty test tube.

Figure 7 shows the PSD for the vibration signals obtained from sensors 1 and 2 when the plastic tube was empty and the PSD of their respective ICA extracted components. ICA has not made a noticeable difference in the recorded vibration signals. A reason for this could be that sensor 2 had been placed close to the vice and thus the vibration it has received had been dampened significantly. The experiment will be repeated in future with sensor 2 placed closer to sensor 1 to reduce this effect.

Figures 8 and 9 show the averaged vibration signals recorded from sensors 1 and 2, when the tube was filled with Methanol. The Methanol signals are shown as an example of the type of signals analysed. A feature of ICA is that, an extracted component can be inverted in relation to its original signal and its magnitude can be changed.
This is the case for the ICA extracted components shown in Figure 8.
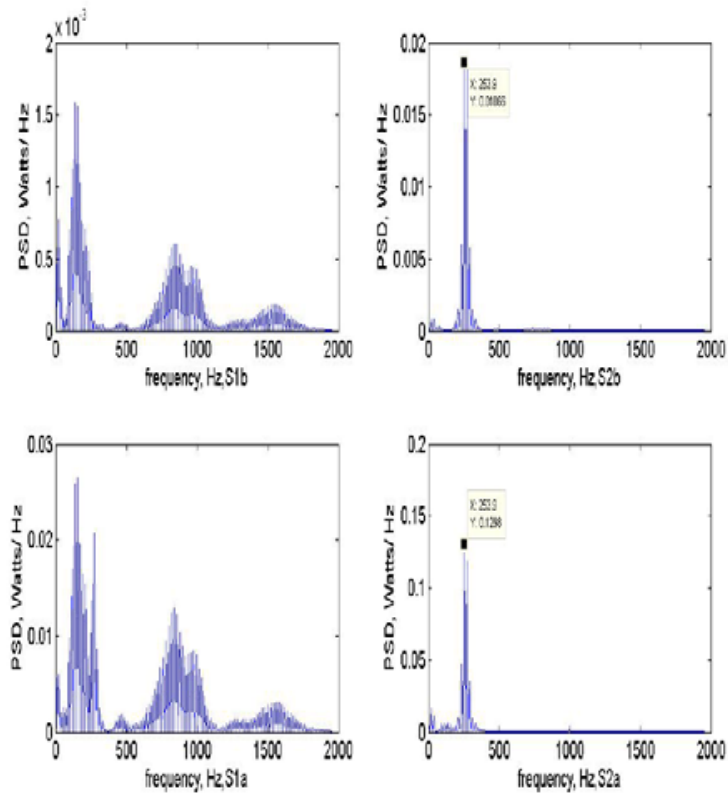

Fig. 7. Power spectral density obtained for sensor 1 before ICA (S1b) and after ICA (S1a) and Power spectral density obtained for sensor 2 before ICA (S2b) and after ICA (S2a), when the test tube was empty.
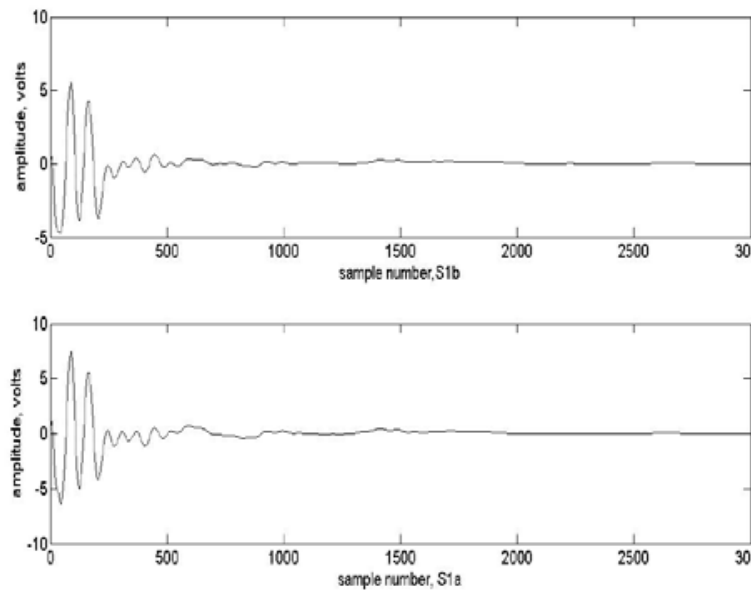

Fig. 8. The averaged vibration signal recorded from sensor 1 before ICA (top) and its corresponding ICA component (bottom)for the test tube filled with Methanol.

The 20 abutted vibration signals and their corresponding ICA components for Methanol are shown in Figures 10 and 11. 

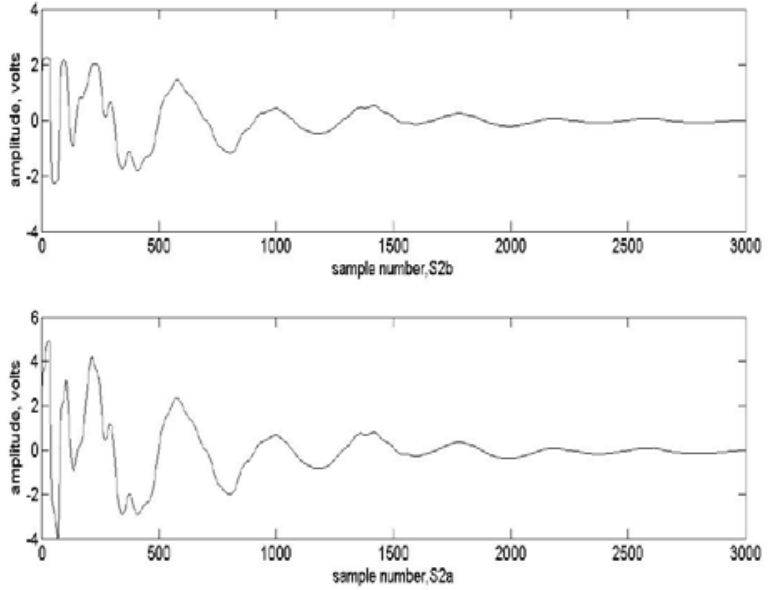

Fig. 9. The averaged vibration signal recorded from sensor 2 before ICA (top) and its corresponding ICA component (bottom) for the test tube filled with Methanol.
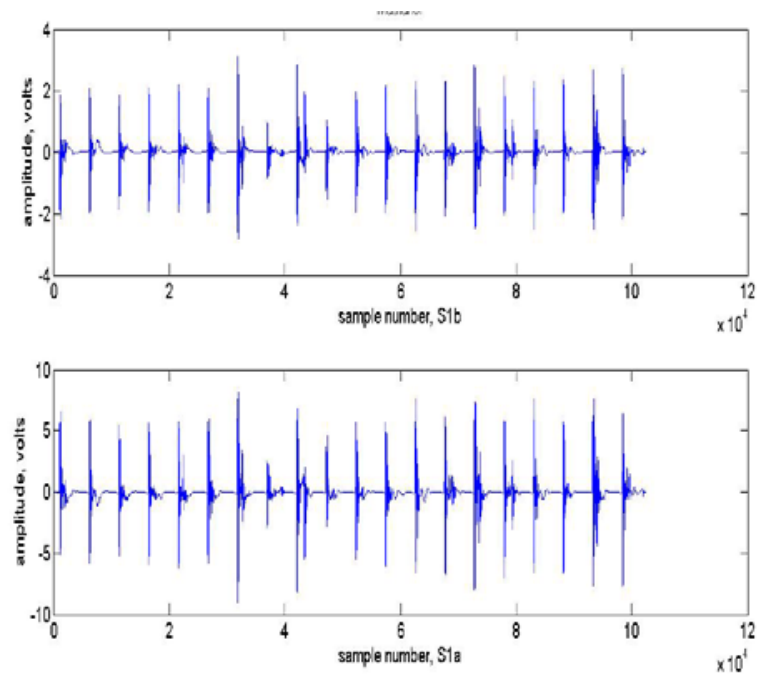

Fig. 10. Twenty abutted vibration signals recorded from sensor 1 before ICA (top) and the corresponding ICA component (bottom) for the test tube filled with Methanol.

As an example, the PSDs associated with the vibration signals from the two sensors, when the test tube was filled with Methanol are shown in Figure 12. The PSDs of the ICA extracted components when the test tube was empty and PSDs when it individually contained the three liquids were compared. This indicated the frequency components in the region of 1000 to $2000 \mathrm{~Hz}$ have greatest difference in the PSDs of the ICA extracted component for the tube and three liquids. Therefore, it was considered that this frequency range had the most relevant vibration information about the objects being tested. The PSD of ICA extracted component for the empty tube had a peak at $1553 \mathrm{~Hz}$ in this frequency region.
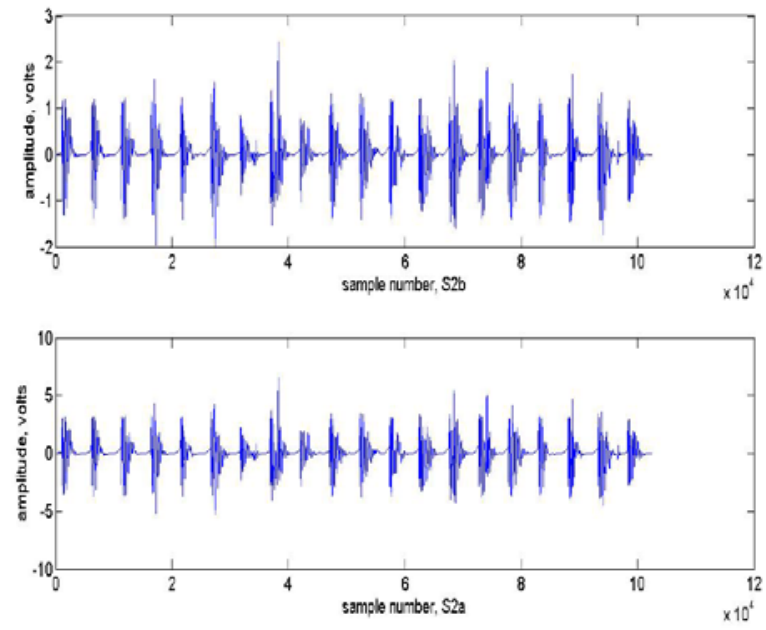

Fig. 11. Twenty abutted vibration signals recorded from sensor 2 before ICA (top) and the corresponding ICA component (bottom) for the test tube filled with Methanol.
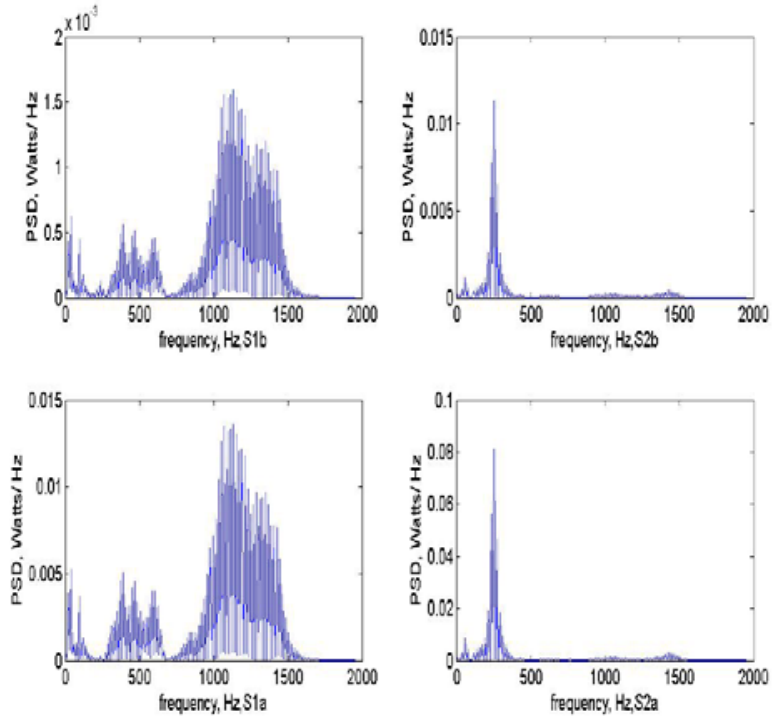

Fig. 12. Power spectral densityobtained for sensor 1 before ICA (S1b) and after ICA (S1a) and power spectral density obtained for sensor 2 before ICA (S2b) and after ICA (S2a), when the test tube was filled with Methanol.

Table 1 indicates the densities of the three liquid liquids used in the study (i.e. Methanol, Water and Chloroform). For each liquid, the frequency that the highest peak occurred in the PSD of sensor 1 , (following ICA) is also shown.

The vibration frequency for each liquid (shown in Table 1) was subtracted from the vibration frequency peak (in the 1000 to $2000 \mathrm{~Hz}$ region) obtained from sensor 1 when the test tube was empty (ie. $1553 \mathrm{~Hz}$ ). This resulted in vibration frequency differences that indicated the effect of the liquid contained in the test tube on the measured vibration. A plot of the resulting vibration frequency differences against the liquids' densities is provided in 
Figure 13.This indicates that as the density of the liquids increases the measured frequency difference increases.

Table 1 The liquid parameters

\begin{tabular}{|c|c|c|c|}
\hline Liquid & $\begin{array}{c}\text { Density } \\
\mathbf{( k g / m}^{\mathbf{3}} \text { ) }\end{array}$ & $\begin{array}{c}\text { Sensor-1 } \\
\text { Extracted } \\
\text { Vibration } \\
\text { Frequency } \\
\text { (Hz) }\end{array}$ & $\begin{array}{c}\text { Vibration } \\
\text { frequency } \\
\text { difference } \\
\text { (Hz) }\end{array}$ \\
\hline Methanol & 791.8 & 1133 & 420 \\
\hline Water & 1000 & 1094 & 459 \\
\hline Chloroform & 1489 & 605 & 948 \\
\hline
\end{tabular}

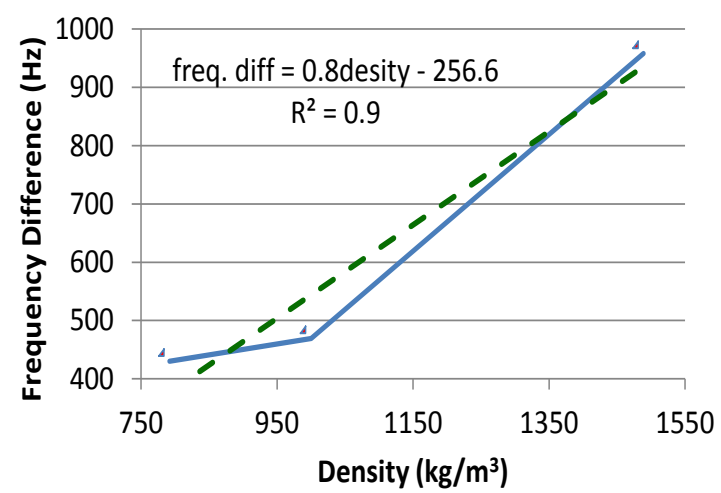

Fig. 13. Plot of vibration frequency difference against the three liquids' densities.

This finding indicatesthat there is a relationship between frequency difference and the liquid density. Desnser liquids have a greater effect on the tube's vibration. In Figure 13, this relationship is approximated to a linear relationship by introducing the best fit through the points, giving the first order relationship equation

$$
\text { frequencydifference }=0.8 \text { density }-256.6
$$

This equation can then be expressed in term of the liquids' densities as,

$$
\text { liquid density }=\frac{\text { frequency difference }+256.6}{0.8}
$$

The experiment needs to be repeated for a larger number of liquids with a greater range of densities in order to establish whether the density-frequency difference relation is linear (as was considered in this study or it is nonlinear. Another area for further investigation is the manner of vibraion of the the liquid within the tube in relation to the tube's vibration. Finally, more critical investigations are needed to establish the effectivenees of ICA to analyse vibration signals.

\section{CONCLUSIONS}

This study investigated the use of vibration analysis and independent component analysis (ICA) to assess the densities of three liquids in a test tube. There was a relationship between the densities of the liquids and the vibration frequency of the ICA extracted frequency components.

This is a preliminary study and a more detailed investigation is required to assess the effectiveness of ICA in extracting different components of the vibration signals and to determine the nature of the relationship (i.e. linear or nonlinear) between the vibration frequency and the liquid densities.

\section{REFERENCES}

[1] S.A. Neild, P.D. McFadden, M.S. Williams, Engineering Structures, 2003,vol.25, pages 713-728.

[2] T.C. Tsai and Y. Z. Wang, Journal of Sound and Vibration", 1996, vol. 192, no.3, pages 607-620.

[3] S. Ebersbach, Z. Peng, Expert Systems with Applications, 2008, vol. 34, pages 291-299.

[4] A. Hyva“rinen, E. Oja, Neural Networks, 2000, vol.13, issues 4-5, pages 411-430.

[5] J. V. Stone, Trends in Cognitive Sciences. 2002, vol.6, issue 2, pages 59-64.

[6] A. J.Bell and T.I. Sejnowski, Neural Computation 1995, vol. 7, pages 1129-1159.

[7] M. J. Roan, J. G. Erling and L. H. Sibul, Mechanical Systems and Signal Processing 2002, vol. 16, no.5, pages 719-740.

[8] J. Antoni, Mechanical Systems and Signal Processing, 2005,vol.19,pages 1166-1180.

[9] G. Kerschen, F. Poncelet, J.-C. Golinval, Mechanical Systems and Signal Processing, 2007, vol. 21, pages1561-1575.

[10] G. Gelle, M. Colas and G. Delaunay, Mechanical Systems and Signal Processing, 2000,vol. 14, no.3, pages 427-442. 